\title{
Synthesis and characterization of super-microporous material with enhanced hydrothermal stability
}

\author{
SHUJIE WU, KE SONG, JINGQI GUAN and QIUBIN KAN* \\ College of Chemistry, Jilin University, Changchun 130023, P.R. China
}

MS received 9 July 2009; revised 18 November 2009

\begin{abstract}
Super-microporouos silicon material with high hydrothermal stability denoted as MCM-41-T has been prepared from mesoporous MCM-41 by high temperature treatment. The structural and chemical property of MCM-41-T has been characterized by X-ray diffraction, transmission electron microscopy, $\mathrm{N}_{2}$ adsorption-desorption, infrared spectroscopy and ${ }^{29} \mathrm{Si}$ MAS NMR. The characteristic results show that Si-OH groups are forced to condense by high temperature treatment, and the pore size of MCM-41-T is around $1.5 \mathrm{~nm}$ in the super-microporous range. Compared with the original material MCM-41, the hydrothermal stability of MCM-41-T has been significantly enhanced.
\end{abstract}

Keywords. MCM-41; mesoporous; super-microporous; hydrothermal stability.

\section{Introduction}

Since the discovery of M41S by Mobil scientists in 1992 (Kresge et al 1992), mesoporous materials have attracted considerable attention for their potential application as catalysts and supports. Until now, numbers of mesophase materials with wide range have been synthesized, such as SBA-15 (Zhao et al 1998), HMS (Tanev and Pinnavaia 1995), MSU (Bagshaw et al 1995) and KIT-1 (Ryoo et al 1996) series. The pore size of mesoporous materials can be controlled by choosing surfactants with different carbon chain lengths, in which the smallest pore size obtained is about $2 \mathrm{~nm}$. As a catalyst or support carrier, however, the pore size of microporous zeolite is too small to diffuse organic reactant in the pore channel, whereas the pore size of mesoporous materials is too large to possess sharp- and size-selective properties in catalysed reactions. Therefore, super-microporous materials possessing uniform size pores in the $1.0-2.0 \mathrm{~nm}$ size range may be better suited for the applications. In addition, super-microporous materials are very important in industrial applications because they bridge the gap of microporous zeolites and mesoporous materials. However, until now, much less attention has been devoted to the synthesis of super-microporous.

Using Gemini surfactant, sodium silica as precursor, through $\mathrm{S}^{+} \mathrm{I}^{-}$routine, highly ordered super-microporous silica was synthesized by Wang et al (2007). Ordered supermicroporous silica with pore size of $1.96 \mathrm{~nm}$ was synthesized from a double-chain surfactant by Ryoo et al (2001). ö-hydroxy-bolaform as surfactants, highly

\footnotetext{
*Author for correspondence (qkan@mail.jlu.edu.cn)
}

ordered super-microporous silica with pore size of 1.4$2.0 \mathrm{~nm}$ has been synthesized by Bagshaw and Hayman (2000). A series of lamellar super-microporous silica with pore size of $1.2-1.5 \mathrm{~nm}$ were synthesized by nanocasting with ionic liquids (Zhou and Antonietti 2003). A nonionic semifluorinated surfactant with short fluorocarbon chains used as a template, ordered hexagonal supermicroprouos silica material (JLU-14 $4_{\mathrm{L}}$ ) has been synthesized at low temperature $\left(0\right.$ to $\left.-20^{\circ} \mathrm{C}\right)$ (Di et al 2006). Tetraalkoxysilane (TMOS) as silica source, 10-alkyl trimethylammonium bromide $\left(\mathrm{C}_{10} \mathrm{TABr}\right)$ used as surfactant, super-microporous silica spheres has been synthesized (Yano and Fukushima 2003). Besides silica materials, some super-microporous materials with metal element (such as alumina, zinc, zirconium, tin, niobium, chromium etc) or organic groups have also been synthesized (Chen et al 1993; Huang et al 1999; Serrano et al 2000; Mcinall et al 2001; Polverejan et al 2002; Casagrande et al 2004; Hiyoshi et al 2004; Ren et al 2004; Shpeizer et al 2005; Xia and Mokaya 2005; Zhao et al 2005; Shpeizer et al 2006; Wang et al 2006).

Up to now, the synthesis of super-microporous materials is one step using special surfactant reagent. Except for pore distribution, the other physical chemistry characters of super-microporous materials have no difference with mesoporous materials. Some disadvantages of mesoporous silicon materials such as poor hydrothermal stability also exist in conventional super-microporous silicon materials. In addition, the thermal stability of mesoporous materials is poor. Under $850^{\circ} \mathrm{C}$ in air for $2 \mathrm{~h}$, the mesoporous pore structure was destroyed completely (Chen et al 1993), therefore the mesoporous materials cannot be treated by high temperature directly. However, it is well 
known that high temperature can make the framework of pore materials with small pore size more condensed to shrink into super-microporous range and hence enhance the hydrothermal stability. Herein we make sucrose dip into the pore of pure silica MCM-41 to keep the pore structure, and then treat it with high temperature under nitrogen condition. After removing the carbon filled in pore channel, super-microporous silica with high hydrothermal stability is obtained.

\section{Experiment}

\subsection{Synthesis of MCM-41-T}

Purely siliceous MCM-41 was prepared according to the procedure reported in the literature (Wang et al 2006). $1.25 \mathrm{~g}$ of sucrose was dissolved into a solution containing $4.1 \mathrm{ml}$ of distilled water and $1 \mathrm{ml}$ of $2 \mathrm{M}$ sulphuric acid solution, then $1 \mathrm{~g}$ calcined MCM-41 was added. The mixture was stirred for $24 \mathrm{~h}$ under room temperature. Afterwards the mixture was placed in a drying oven at $100^{\circ} \mathrm{C}$ for $6 \mathrm{~h}$, and then the temperature was increased up to $160^{\circ} \mathrm{C}$ to further dry for $6 \mathrm{~h}$ under nitrogen condition. After cooling down to room temperature, the sample was treated again at 100 and $160^{\circ} \mathrm{C}$ after the addition of $0.8 \mathrm{~g}$ of sucrose, $0.5 \mathrm{ml}$ of $2 \mathrm{M}$ sulphuric acid and $4.55 \mathrm{~g}$ of distilled water. The carbonization was completed by high temperature calcination at $900^{\circ} \mathrm{C}$ for $7 \mathrm{~h}$ under nitrogen condition. After cooling down to room temperature, the sample was further calcined under air at $650^{\circ} \mathrm{C}$ for $6 \mathrm{~h}$ to obtain the final catalyst MCM-41-T.

\subsection{Hydrothermal stability studies}

$0.6 \mathrm{~g} \mathrm{MCM}-41$ (or MCM-41-T) and $60 \mathrm{ml}$ water were added in a flask with reflux device. The mixture was heated to boiling point and kept boiling condition for needed time, then filtered. The resulting sample was dried at $110^{\circ} \mathrm{C}$ for characterization.

\subsection{Characterization}

Powder X-ray diffraction patterns were recorded on a Shimadzu XRD-6000 diffractometer system equipped with Ni-filtered $\mathrm{CuK} \alpha$ radiation (work at $40 \mathrm{kV}, 30 \mathrm{~mA}$, wavelength $\lambda=0 \cdot 15418 \mathrm{~nm})$. The d-bound of angle $(2 \theta)$ is from 1.5 to 10 , at a scanning speed of $1 \mathrm{~min}^{-1} . \mathrm{N}_{2}$ adsorption-desorption isotherms at $77 \mathrm{~K}$ was recorded with a Micromeritics ASAP 2010 system. Before measurement, the sample was out gassed at $250^{\circ} \mathrm{C}$ for $3 \mathrm{~h}$. The specific surface area was calculated by Brunauer-EmmettTeller (BET) method. The pore size distributions were calculated from adsorption branches of nitrogen isotherms using Barrett-Joyner-Halenda (BJH) method. The total pore volume was taken at relative pressure of 0.989 . IR spectra were obtained using a Nicolet Avatar 370Dtgs spectroscopy, the samples were mixed with $\mathrm{KBr}$ and pressed as pellets. ${ }^{29} \mathrm{Si}$ MAS NMR spectra were measured on a Varian Unity-400 spectrometer.

\section{Result and discussion}

\subsection{XRD and TEM studies}

XRD patterns of the samples before and after high temperature treatment are shown in figure 1. For as-synthesized material MCM-41, three diffraction peaks indexable as (100), (110) and (200) reflections are the characteristic peaks of hexagonal $p 6 \mathrm{~mm}$ structure. However, for the sample calcined at $900^{\circ} \mathrm{C}$ for $7 \mathrm{~h}$ without carbon-filled, no diffraction peak can be observed which suggests that the pore structures are destroyed completely. Nevertheless, for MCM-41 calcined at $900^{\circ} \mathrm{C}$ for $7 \mathrm{~h}$ with carbonfilled, the XRD peak of 100 still exists, indicating that the pore structures have been retained. The result suggests that the carbon filled in the pore has a positive effect on the supporting pore structures. However, there are some changes between the samples before and after high temperature treatment. After high temperature treatment, the site $2 \theta$ of peak 100 increases from 2.53 to $2 \cdot 89$, and the D-spacing 100 shrinks from $3.58 \mathrm{~nm}$ to $3.14 \mathrm{~nm}$, while the diffraction peaks of 110 and 200 disappear, indicating that the framework of mesoporous has some contraction. We deem that pore structure still reserves after high temperature treatment, but the long-ordered channels have some break. This conclusion can be proved by transmission electron microscope (TEM) image (figure 1). Disordered

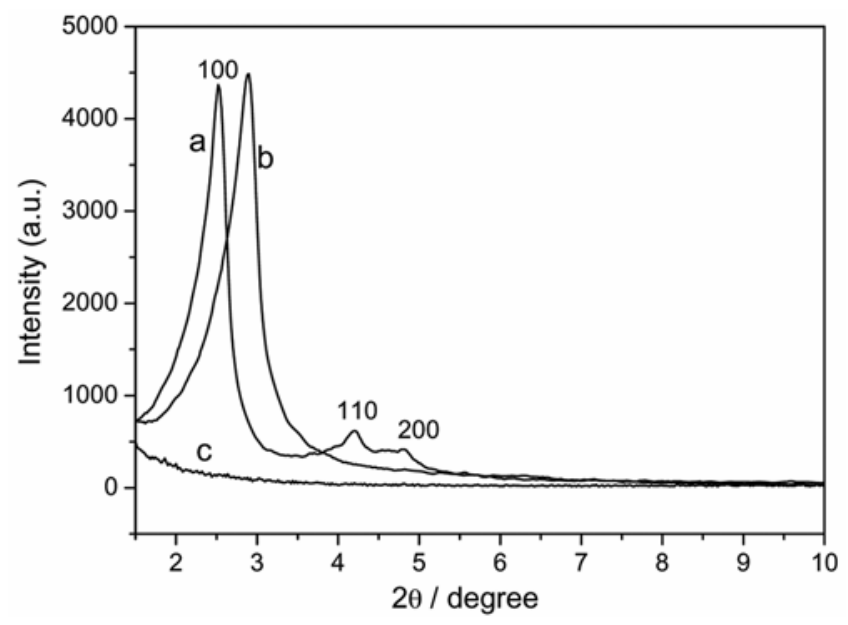

Figure 1. XRD patterns of different samples: (a) calcined MCM-41; (b) calcined MCM-41 in $900^{\circ} \mathrm{C}$ for $7 \mathrm{~h}$ with carbon filled denoted as MCM-41-T; (c) calcined MCM-41 in $900^{\circ} \mathrm{C}$ for $7 \mathrm{~h}$ without carbon filled. 
pores can be observed clearly. Since the pore structure of MCM-41 is two-dimension unconnected, under high temperature, both the silicon framework and carbon filled in the pore shrink acutely, and therefore, the pore can be saved but the long-ordered channel has been destroyed.

\section{$3.2 \mathrm{~N}_{2}$ adsorption-desorption studies}

$\mathrm{N}_{2}$ adsorption-desorption isotherms of the samples before and after high temperature treatment are shown in figure 2. Before high temperature treatment, typical type IV curve and sharp hysteresis curves at relative pressure from 0.20 to 0.40 correspond to mesoporous structures. The pore size obtained from BJH methods is $2.45 \mathrm{~nm}$ corresponding to MCM-41. After high temperature treatment, the frameworks of MCM-41 shrink very acutely and the pore size decreases sharply from mesoporous range to super-microporous range. Pore diameter as calculated by the BJH method is around $1.53 \mathrm{~nm}$ for MCM41-T. The $\mathrm{N}_{2}$ adsorption-desorption isotherms change from type IV curve to mixed type I and IV curves after high temperature treatment. In addition, the pore volume calculated by BET method also decreases evidently from 1.20 to $0.47 \mathrm{~cm}^{3} / \mathrm{g}$. Compared with MCM-41, both the surface area and pore volume of MCM-41-T decrease.

\subsection{IR studies}

Figure 3 shows IR spectra of MCM-41 and MCM-41-T. The bands at 1217 and $1094 \mathrm{~cm}^{-1}$ can be assigned to Si$\mathrm{O}-\mathrm{Si}$ dissymmetry stretching vibration, while the bond at $960 \mathrm{~cm}^{-1}$ can be due to the $\mathrm{Si}-\mathrm{OH}$ stretching vibration (Huang et al 1999). In addition, $806 \mathrm{~cm}^{-1}$ can be ascribed to symmetry stretching vibration, whereas the bond at $460 \mathrm{~cm}^{-1}$ can be attributed to the bending vibration of the

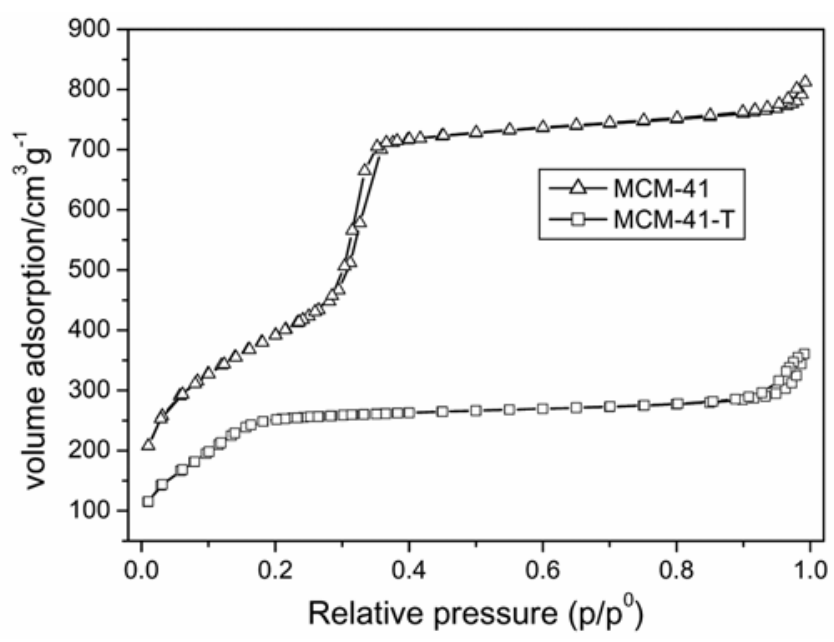

Figure 2. $\mathrm{N}_{2}$ adsoption-desorption isotherms of MCM-41 and MCM-41-T. silica. There is an obvious difference between MCM-41 and MCM-41-T at $960 \mathrm{~cm}^{-1}$. For MCM-41, an obvious vibrational peak at this site can be observed, while for MCM-41-T, the vibrational peak at this wavenumber is not obvious. The reason for the difference is that there are large numbers of $\mathrm{Si}-\mathrm{OH}$ groups at the external of MCM41 framework. After treatment with high temperature, large numbers of $\mathrm{Si}-\mathrm{OH}$ groups are forced to condense, and water molecular is lost from chemical bonds of $\mathrm{Si}-\mathrm{OH}$. Therefore, more Si-O-Si chemical bonds are formed. This conclusion can also be proven by MAS ${ }^{29} \mathrm{Si}$ NMR.

\section{$3.4{ }^{29}$ Si MAS NMR studies}

Figure 4 shows the ${ }^{29} \mathrm{Si}$ MAS NMR spectra of the samples before and after high temperature treatment. Two

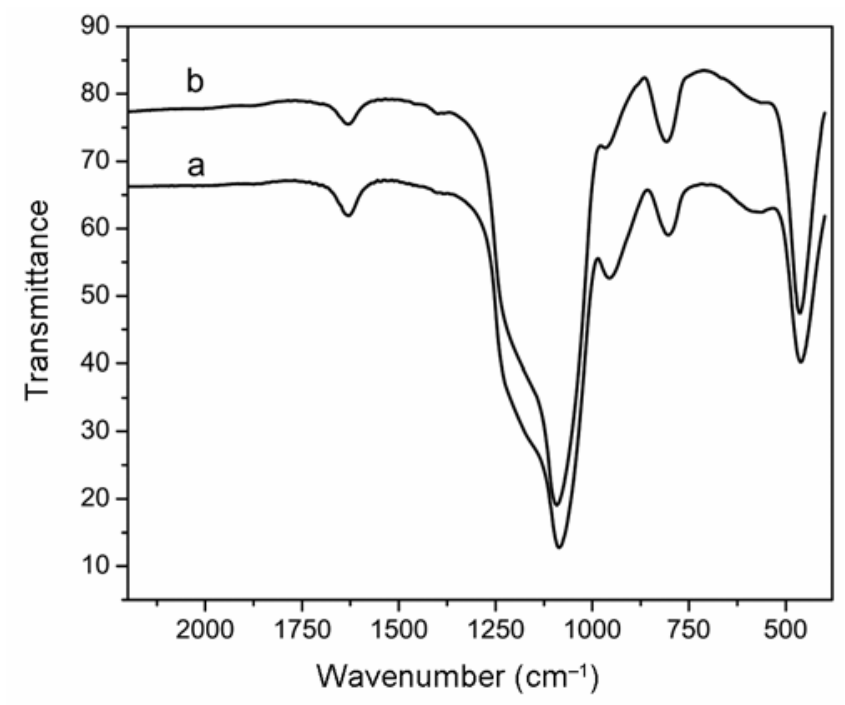

Figure 3. IR spectra of calcined MCM-41(a) and MCM-41$\mathrm{T}(\mathrm{b})$

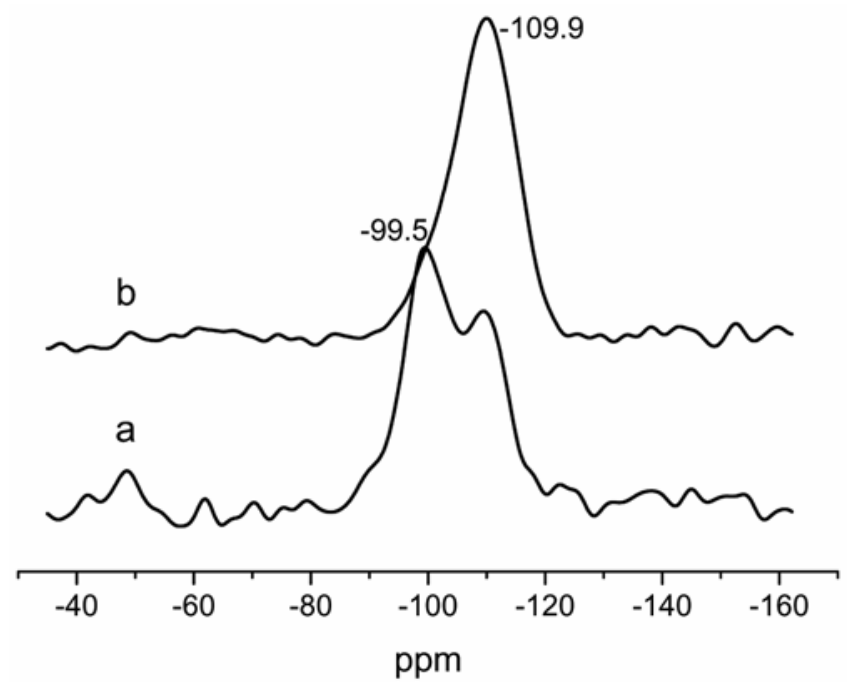

Figure 4. ${ }^{29} \mathrm{Si}$ MAS NMR spectra of (a) MCM-41 and (b) MCM-41-T 
peaks at -99.5 and $-109.9 \mathrm{ppm}$, correspond to $(\mathrm{SiO})_{3}-\mathrm{Si}-$ $\mathrm{OH}\left(\mathrm{Q}^{3}\right)$ and $(\mathrm{SiO})_{4}-\mathrm{Si}\left(\mathrm{Q}^{4}\right)$, respectively (Chen et al 1993). Compared with MCM-41, the ${ }^{29} \mathrm{Si}$ NMR spectrum of MCM-41-T shows a significant increase in the NMR peak ratio between $\mathrm{Si}(-\mathrm{OSi})_{4}$ tetrahedral silicon atoms and $\mathrm{Si}(-\mathrm{OSi})_{3}(-\mathrm{OH})$ silicon atoms $\left(\mathrm{Q}^{4} / \mathrm{Q}^{3}\right)$ due to the high temperature treatment. The ratio of $\mathrm{Q}^{4} / \mathrm{Q}^{3}$ is 0.69 for MCM-41 and 6.1 for MCM-41-T, respectively. It is reasonable to believe that excess $\mathrm{Si}-\mathrm{OH}$ groups are forced to condense under high temperature treatment. Less $\mathrm{Si}-\mathrm{OH}$ groups can hardly be attacked by hydrolysis in hot water, thus the hydrothermal stability can be enhanced by high temperature treatment.

\subsection{Hydrothermal stability studies}

The results of hydrothermal stability studies are shown in figure 5. For MCM-41, after refluxed in boiling water for
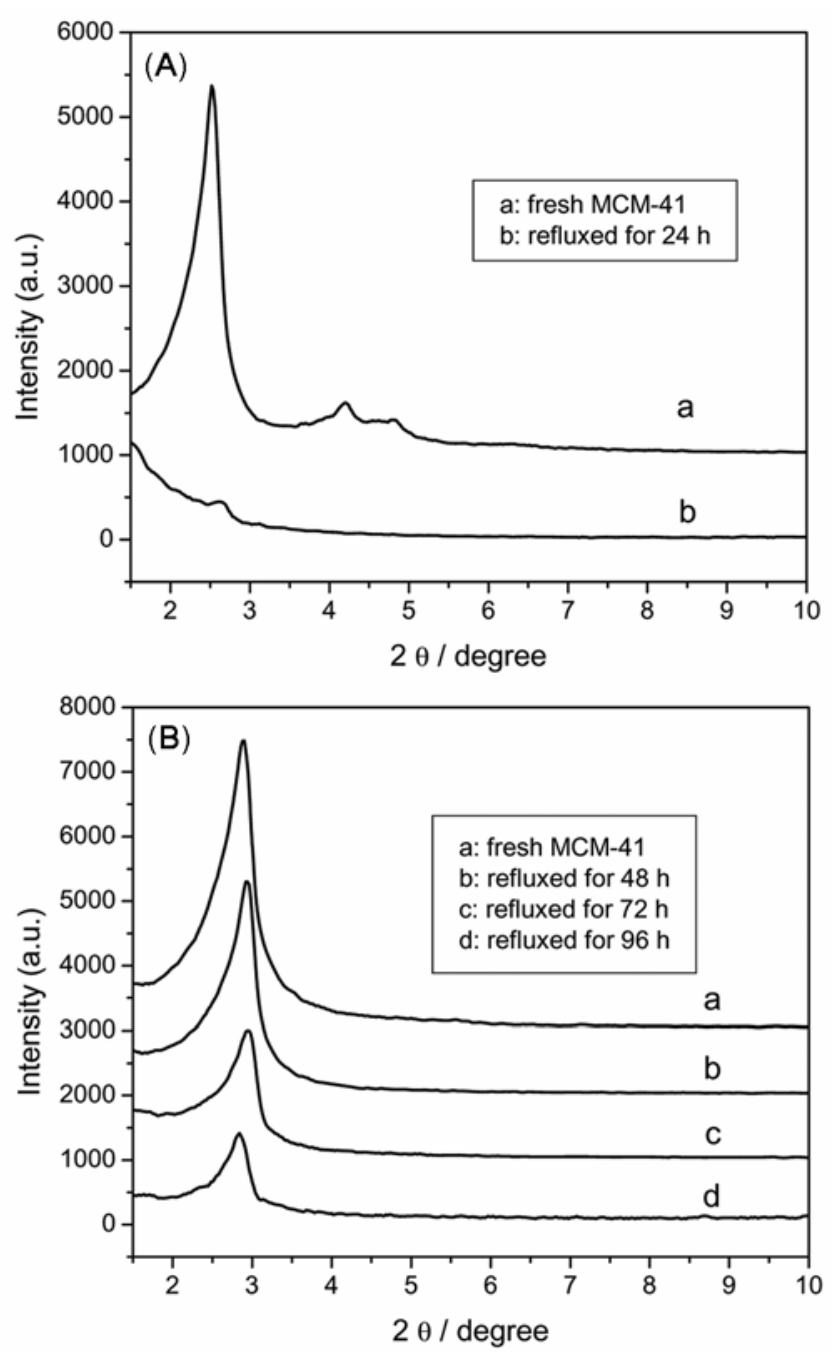

Figure 5. XRD patterns of MCM-41 (A) and MCM-41-T (B) refluxed in boiling water for different times.
$24 \mathrm{~h}$, almost no diffraction peak can be observed suggesting that the pore structures are completely destroyed. However for MCM-41-T, after refluxed in boiling water for $96 \mathrm{~h}$, the 100 diffraction peak still exists indicating that pore structure has been saved. Thin pore walls and more silanol groups make MCM-41 cannot counteract the attack of boiled water. Two reasons lead MCM-41-T to enhanced hydrothermal stability as compared with conventional mesoporous material MCM-41. One is more concentration framework (less silanol groups, high $\mathrm{Q}^{4} / \mathrm{Q}^{3}$ ratio), and another is thicker pore wall. The pore thickness was increased slightly (only $0.32 \mathrm{~nm}$ ), but the $\mathrm{Q}^{4} / \mathrm{Q}^{3}$ ratio increases obviously from 0.69 to $6 \cdot 1$. Therefore the decrease of silanol group is the primary reason for enhancing hydrothermal stability.

\section{Conclusions}

Up to now, almost all methods synthesized of silicon supermicroporous materials is one-step used special template reagent, and the character is similar with conventional mesoporous material except lesser pore size. We synthesized supermicroporous molecular sieves with high hydrothermal stability by high temperature treatment from meosporous molecular sieve MCM-41 for the first time. After high temperature treatment, the pore size of MCM-41 shrinks into super-microporous range. At the same time, the $\mathrm{Si}-\mathrm{OH}$ groups at the surface of framework condense acutely, and $\mathrm{Q}^{4} / \mathrm{Q}^{3}$ ratio increases considerably. Compared with MCM-41, the hydrothermal stability of MCM-41-T has been enhanced remarkably. The special character of high hydrothermal stability may make the MCM-41-T have extensive use in chemistry industry.

\section{References}

Bagshaw S A, Prouzet E and Pinnavaia T J 1995 Science 269 1242

Bagshaw S A and Hayman A R 2000 Chem. Commun. 533

Casagrande M, Storaro L, Lenarda M, Gersich J, Stievano L, Wagner F E and Montanari T 2004 J. Mater. Chem. 14 1010

Chen C Y, Li H X and Davis M E 1993 Microporous Mater. 2 17

Di Y, Meng X, Wang L, Li S and Xiao F S 2006 Langmuir 22 3068

Hiyoshi M, Lee B, Lu D, Hara M, Kondo J N and Domen K 2004 Catal. Lett. 98181

Huang Y, Jiang Z and Schwieger W 1999 Chem. Mater. 11 1210

Kresge C T, Leonowicz M E, Roth W J, Vartuli J C and Beck J S 1992 Nature 359710

Mcinall M D, Scott J, Mercier L and Kooyman P J 2001 Chem. Commun. 2282

Polverejan M, Liu Y and Pinnavaia T J 2002 Chem. Mater. 14 2283

Ren T Z, Yuan Z Y and Su B L 2004 Chem. Commun. 2730 
Ryoo R, Kim J M, Ko C H and Shin C H 1996 J. Phys. Chem. 10017718

Ryoo R, Park I S, Jun S, Lee C W, Kruk M and Jaroniec M $2001 \mathrm{~J}$. Am. Chem. Soc. 1231650

Serrano D P, Aguado J, Escola J M and Garagorri E 2000 Chem. Commun. 2041

Shpeizer B G, Clearfoeld A and Heising J M 2005 Chem. Commun. 2396

Shpeizer B G, Bakhmutov V I and Clearfield A 2006 Micro. Meso. Mater. 9081

Tanev P T and Pinnavaia T J 1995 Science 267865
Wang K, Lin Y, Morris M A and Holmes J D 2006 J. Mater. Chem. 164051

Wang R L, Han S H, Hou W G, Sun L X, Zhao J and Wang Y S 2007 J. Phys. Chem. C111 10955

Xia Y and Mokaya R 2005 Micro. Meso. Mater. 86231

Yano K and Fukushima Y 2003 J. Mater. Chem. 132577

Zhao D, Feng J, Huo Q, Melosh N, Fredrickson G H, Chmelka B F and Stucky G D 1998 Science 279548

Zhao G L, Yuan Z Y and Chen T H 2005 Mater. Res. Bull. 40 1922

Zhou Y and Antonietti M 2003 Adv. Mater. 151452 